\title{
Non surgical approaches in treatment of OSF
}

\author{
Usha Dayanarayana ${ }^{1}$, Nagabhushana Doggalli ${ }^{2}$, Karthikeya Patil $^{3}$, Jai Shankar ${ }^{4}$, \\ Mahesh K.P ${ }^{5}$, Sanjay. ${ }^{6}$ \\ ${ }^{I}$ (Department of Oral Medicine and Radiology, JSS Dental College and Hospital, Mysore, Karnataka, India)
}

\begin{abstract}
Oral submucous fibrosis (OSF) is often mocked as a condition which is easy to diagnose, but extremely difficult to manage. It is a chronic, debilitating disease of the oral cavity. Though it has a global distribution, it is predominantly a disease of the Indian subcontinent. A standard treatment to manage this condition is not yet available. This article aims at enumerating various treatment modalities of OSF.
\end{abstract}

Key words: Management of OSF, Oral submucous fibrosis, Premalignant condition,

\section{Introduction}

Oral submucous fibrosis (OSF) is a chronic, progressive, scarring disease. It is characterized by blanching, stiffening and fibrosis of any area of the oral cavity. It results in loss of tissue mobility, increases rigidity and an eventual inability to open the mouth.[1]

The widely accepted definition of OSMF was proposed by Pindborg and Sirsat in 1966. Oral submucous fibrosis is defined as "An insidious chronic disease affecting any part of the oral cavity and sometimes the pharynx. It is always associated with juxta-epithelial inflammatory reaction followed by fibroelastic changes of the lamina propria with epithelial atrophy leading to stiffness of the oral mucosa and causing trismus and inability to speak".[2]

The disease is exclusively reported in Indian population and in South East Asia, but the cases have been reported worldwide like Kenya, China, UK, Saudi Arabia and other parts of the world where Asians have migrated and are migrating.[3]Worldwide, approximately 2.5 million people are affected, with most cases concentrated on the Indian subcontinent, especially southern India.[4] Male-female ratio of 9.9:1[5]

Etiology of OSF is not fully understood, although various factors have been implicated in the development. Factors included are arecanut chewing, ingestion of chillies, genetic, immunologic processes, autoimmunity, hypersensitivity, nutritional deficiency, salivary coagulopathic factors and increased lysyl oxidase levels etc.

The most commonly involved site is buccal mucosa, followed by palate, retromolar region, faucial pillars and pharynx[6] and in some cases can even involve the pharynx, larynx, or the esophagus.[7] It causes a stiffening of the oral mucosa due to a fibrotic change in the mucosa and its connective tissue which then restricts the mouth opening.[8]

It has been linked with an increased risk of malignancy and thus is considered as a pre-malignant condition. [9]

The disease has a variety of presentations ranging from, excessive salivation, burning sensation, lacking gustatory sensation and limitation of mouth opening leading to difficulty in chewing, swallowing, articulation and poor oral hygiene and its complications.[9]

There is no definite treatment of OSF. The main aim of treatment is to relieve the symptoms and improve the mouth opening.

The various treatment modalities are mainly medical, surgical, or a combination of both. The treatment depends on the level of clinical involvement. At a very early stage, cessation of the habit is adequate. Medical/surgical treatment is necessary for moderate to severe cases. Surgical treatment is the method of choice in patients with marked limitation of mouth opening or in patients not responding to the conservative management.[10]

\section{Management of OSF can be divided into}

- Preventive measures - Discontinuation of Habit and Counseling

- Nutritional support

- Physio therapy:

○ Kneading

- Muscle stretching exercises

- Heat-Short wave/Micro wave diathermy

- Medical management

○ Allopathic 


\title{
- Ultrasound
}

- Alternate medicines

\subsection{Preventive measures - Discontinuation of Habit and Counseling}

Reduction or even elimination of the habit of areca nut chewing is an important preventive measure. They should also be instructed to minimize consumption of spicy foods and maintain proper oral hygiene. They should be explained about the condition and its probable malignant potential and counseling should be given for de-addiction. [11,12]

Nicotine Replacement Therapy is supplied as a gum, skin patch, nasal spray, or inhaler. It works by providing a substitute source of nicotine, without the other harmful components of cigarette smoke. Nicotine replacement therapy can reduce or eliminate the withdrawal symptoms many have when they quit smoking.

Zyban (bupropion hydrochloride) and Chantix (varenicline) are non-nicotine-containing medications available by prescription. Zyban reduces the symptoms of nicotine withdrawal by acting on chemicals in the brain related to nicotine cravings. Zyban is also prescribed under another name 'Wellbutrin' to treat depression. Chantix lessens pleasure from smoking and reduces symptoms of withdrawal.

\subsection{Nutritional support}

The rationale of giving nutrients in OSF patients is to correct deficiency states and promote normal cellular processes present in health that help to protect against adverse events including carcinogenesis.[11]

- Supplementing the diet with foods rich in vitamins A, B complex, and C and iron

- Advice green leafy vegetables, red tomatoes, fresh fruits.

- Advice Green tea

- High protein diet

Vitamin A plays a major role in the epithelial differentiation, in mucous secretary and keratinisation tissues and as well as in adequate concentration it delays, slows, arrests or even reverse the progress of premalignant cells to cells with invasive malignant potential.

A study was done to evaluate effectiveness of Micronutrients in 64 patients with oral submucous fibrosis by Nidhi Thakur. Mouth opening in patients showed significant improvement at the end of 6 weeks as compared to the initial mouth opening.[13]

\subsection{Physiotherapy}

Physiotherapy in the form of forceful mouth opening, and heat has been tried.

Kneading is an effective form of massage therapy in improving the elasticity of fibrous tissues and mobilizing scar tissues. The gentle soft tissue manipulation is extensively used in physiotherapy for improving their extensibility.The decrease in Temporo-mandibular joint mobility is mobilized by forced passive mevements \& manipulations to permit more mouth opening.[14]

Muscle stretching exercises for the mouth may be helpful to prevent further restriction of mouth movements and to prevent relapse. This can be performed using mouth gag, acrylic surgical stent, ballooning of mouth, hot water gargling, inter positioning spatula between the teeth and adding a new spatula every 5-10 days. This is thought to put pressure on fibrous bands.[12]

It is advocated to use the oral stents over a period of 6 months. It should be removed only while during meals and at bedtime otherwise the patient should wear it all day.

Heat -Short wave/Micro wave diathermy: Heat has been used in the form of lukewarm water, hot rinses or selective deep heating therapies like short wave or micro wave diathermy.[15] It acts by physiofbrinolysis of bands. Microwave diathermy seems superior to short wave, because selective heating of juxta epithelial connective tissue, thereby limiting the area treated.[12]

Gupta DS et al used microwave diathermy at $2450 \mathrm{MC} / \mathrm{s}$ daily for 20 mins at each site of the lesion with 20 to 25 watts of energy, 15 sittings were given and reported that the use of microwave diathermy of great significance for moderately advanced stages but in very advanced cases results were very poor.[16]

\author{
2.4 Medical management \\ Corticosteroids \\ Peripheral Vasodilators \\ Placental extracts \\ Recombinant Human Interferon Gamma ( $\gamma$-IFN) \\ Enzymes \\ Alternative Medicine \\ - Antioxidents, Immunised milk, Turmeric
}


Combination regimen

\subsubsection{Corticosteroids}

Glucocorticoids were the first group of drugs to be used in the treatment of OSMF patients. Due to its anti inflammatory action, it inhibits the production of inflammatory factors and increases the apoptosis of inflammatory cells. This further inhibits the proliferation of fibroblasts, up-regulation of collagen synthesis and down-regulation of collagenase production. They provide symptomatic relief or is used as an adjunct.[17]

In patients with moderate OSF, weekly submucosal intralesional injections or topical application of steroids may help to prevent further damage. Steroid ointment applied topically helps in cases with ulcers and painful oral mucosa. Its therapeutic effects were mainly anti inflammatory and appeared to have a direct healing action. Steroids act as immunosuppressive agents for prevention or suppression of the fibro productive inflammation found in OSF lesions, thus ameliorating this fibro-collagenous condition.[18]

Short acting drugs: Hydrocortisone intralesional injection $1.5 \mathrm{cc}$ given once a week for a duration of 12 weeks have proven to be beneficial. Systemic corticosteroids were found to be useful in only early and mild cases.

Intermediate acting drugs: Topical triamcinolone acetonide $0.1 \%$ and local injection of triamcinolone acetonide can be used in very early and early cases.

Long acting drugs: Dexamethasone $4 \mathrm{mg}$ intralesional injections, is given biweekly. Also if given in combination with hyaluronidase gives better long term results. Betamethasone is given as $4 \mathrm{mg} / \mathrm{ml}$ intralesional injections biweekly. If given in combination with lycopene or hyaluronidase and vitamin $\mathrm{E}$, it is more effective.

Modalities of corticosteroids used in the treatment

Topical:

- Triamcinalone acetonide $0.1 \%$ (Kenacort)

- Betametasone $-0.5 \%$ (Betnesol)

Systemic :

- Prednisolone $-20 \mathrm{mg} /$ day (Wysolone)

- Dexamethasone - 4mg/day (Dexona)

- Triamcinolone - 12mg/day (Kenacort)

Intralesional :

- Dexametasone - $4 \mathrm{mg} / \mathrm{ml}$ (inj Dexona)

- Triamsinolone $-40 \mathrm{mg} / \mathrm{ml}$ (inj Kenacort)

- Hydrocortisone $-25 \mathrm{mg} / \mathrm{ml}$ (inj Wycort)

\subsubsection{Peripheral Vasodilators}

Pentoxifylline (Trental), a methylxanthine derivative has vasodilating properties. It increases mucosal vascularity, so recommended as an adjunct therapy in management of OSF. It has anti inflammatory properties like altering fibroblast physiology, suppressing leucocyte function and stimulating fibrinolysis. Its immunomodulating actions include neutrophil degranulation, increasing leukocyte adhesion, promotes natural killer cell activity, release of peroxides, production of tumor necrosis factor and inhibits $\mathrm{T}$ and $\mathrm{B}$ cell activation.

Pentoxifylline increases leukocyte chemotaxis, red cell deformability, antithrombin and antiplasmin activities. Pentoxifylline decreases granulocyte adhesion, fibrinogen levels, red cell and platelet aggregation, and whole blood viscosity.[19]

Pentoxifylline $400 \mathrm{mg}$ three times daily, as coated, sustained release tablets have been used as an adjunct therapy resulted in improvement in mouth opening, tongue protrusion, and relief from perioral fibrotic bands.[17]

Nylidrin hydrochloride a peripheral vasodilator affects the tissues in diffuse fibrosis to a noticeable degree by relieving the local ischemic effect and also helps the nutritional and therapeutic substances to reach the affected tissues, because it has been observed that oral submucous fibrosis symptoms include local ischemic effects secondary to local fibrous conditions, hyperkeratosis, and loss of suppleness [20]

\subsubsection{Placental Extracts (Placentrax)}

It is an aqueous extract of human placenta that contains nucleotides, enzymes, vitamins, aminoacids and steroids. They can be divided into four different fractions such as aqueous extracts, lipoidal extracts, immune gamma globulins and tissue coagulants. Only the aqueous extracts of placenta acts as biogenic stimulators in the cellular metabolism assing the absorption of epithelium and increasing the physiological actions of the organs.[21,22]

Aqueous extract of fresh human placenta contains 
a. Enzymes: alkaline and acid phosphatase, glutamic oxaloacetic acid transaminase, glutamic acid and pyruvate transaminase.

b. Nucleotides: ribonucleic acid and deoxy ribonucleic acid, adenosine triphosphate.

c. Vitamins: Vit E, $\mathrm{B}_{1}, \mathrm{~B}_{2}, \mathrm{~B}_{4}, \mathrm{~B}_{6}$, Pantothenic acid, Nicotinic acid, P-amino benzoic acid, Folic acid, Cholin, Ionositol.

d. Amino acids: Alanine, Aspargin, Asparginic Acid, Cystiene, Glutamic Acid, Glycine, Histidine, Leucine, Lysine, Phenyl Alanine, Proline, Serine, Threonine, Tryptophan, Tyrosine, Valine.

e. Steroids: 17- ketosteroids, cholesterin, cholesterol, cholesterin ester.

f. Fatty acids: linoleic acid, Linoleinic acid, oleic acid and palmitic acid.

g. Trace elements: cadium, potassium, calcium, magnesium, Copper, iron, phosphorous and silicone.

Main effects of placental extracts are anti-inflammatory and significant analgesic effect, increase in blood circulation and tissue vascularity, arrest of tissue growth stagnation, metabolic degenerative conditions and lower immune response factor. Placental extracts have found to have $50-100 \mathrm{~K} \mathrm{~A}^{0}$ units of alkaline phophatase, thereby used as local nutrients. [21]

$2 \mathrm{cc}$ of placentrix injection intralesionally at weekly intervals for 10 weeks was found to be superior to cortisone. In resistant cases, surgical excision of fibrotic bands with submucosal placement of fresh human placental grafts was found to be successful.[17] Combination of Dexamethasone, Hyaluronidase and Placental extract gives better results than with a single drug.

\subsubsection{Recombinant Human Interferon Gamma $(\gamma$-IFN)}

$\gamma$-IFN has epithelial regulatory effect, it is a known anti-fibrotic cytokine.[22] The antifibrosis factor caused down regulation of fibroblast proliferation and collagen synthesis, upregulation of antifibrotic cytokine and collagen synthesis in the basal layer of epithelium and lamina propria.[17] Patients treated with $\gamma$-IFN showed improvements in mouth opening,interincisal distance with net gain $8 \pm 4 \mathrm{~mm}(42 \%)$, the range being 4$15 \mathrm{~mm} .23$ Injections of IFN gamma produced a few side effects, which includes head ache, flu like symptoms and myalgia.[21]

Intralesional injection of $\gamma$-IFN (0.01- $10.0 \mathrm{U} / \mathrm{mL}) 3$ times daily for 6 months.[12]

\section{ANTI HELMINTHIC}

\section{Levamisole}

It is an immunomodulator which modifies both cellular and humoral immunity. In OSF it reduces the level of $\operatorname{IgG}$, IgA and IgM thus slow down the chronic inflammatory process. Levamisole 150mg, three times daily for three consecutive days in a week for three alternate weeks or in combination with two capsules of antaoxid daily for six weeks showed symptomatic improvement.

\subsubsection{Enzymes}

Chymotrypsin an endopeptidase, peptide bonds and hydrolyses ester, thus acting as aproteolytic and anti-infammatory agent.[12] Local injection of chymotrypsin have proved to be successful in treating OSF.

Chymotrypsin (5000 IU), twice weekly submucosal injections for 10 weeks

Hyaluronidase reduces burning sensation and trismus. It acts by breaking down hyaluronic acid, lowers the viscosity of intracellular substances and decreases collagen formation.[24] It acts by spreading or diffusing substance, which modifies the permeability through the hydrolysis of hyaluronic acid, a polysaccharide found in the intercellular ground substance of the connective tissue; it temporarily decreases the viscosity of the cellular cement and promotes diffusion of injected fluids or of localized transudates or exudates thus facilitating its absorption.[16]

The combination of steroids and hyaluronidase shows better long-term results than either agent used alone.

Intralesional injections of 1500 IU of hyaluronidase mixed with $2 \%$ lignocaine twice daily for 10 weeks have been tried and satisfactory results have been obtained in OSF patients who have severe limitation in mouth opening

Collagenase: is a lysosomal enzyme, capable of degrading phosphate esters, proteins, polysaccharides, glycosides and sulphate esters. In a controlled clinical trial, Lin and Lin found that intralesional injections of collagenase resulted not only in significant improvement in mouth opening, but also in a striking reduction of hypersensitivity to spices, sour, cold, and heat. Collagenase treatment is approximately five fold more effective than triamcinolone acetate.

Dose: $2 \mathrm{mg}$ of collagenase materials dissolved in $1 \mathrm{ml}$ of distilled water for injection purposes.

Adverse reactions: Adverse reactions like pain swelling and trismus may be seen after injections of collagenase which is considered to be allergic reaction of this agent. 


\section{CARDIOVASCULAR DRUGS}

In OSF the deposition of collagen fibres can cause occlusion of blood vessels resulting in restriction of nutrients substances from reaching the affected areas. Cardiovascular drugs are used to improve the circulation and hemorrheology. Pentoxifylline, buflomedil, nylidrin, tea pigments are some of the agents used.[17]

Buflomedil a vasoactive agent, acts on the microcirculation, relieves the ischemic effect and thus helps the nutritional and therapeutic substances reach the affected tissues. It can be used as effective adjunct drug both in early and advanced cases of OSF.[25]

Nylidrin a sympathomtmetic agent, chemically related to the epinephrine-ephedrine series. It produces vasodilation of arterioles of skeletal muscles. Nylidrin in conjunction with conventional therapy (ex: vitamin complexes and corticosteroids) is used to treat effectively OSF in younger individuals and early onset cases. Nylidrin is effective with prolonged action after oral administration as it is immune from attack by monoamine oxidase and catechol-o-methyl transferase.

\section{ANTIOXIDENTS}

Antioxidants act by reducing the free radical reaction that can cause DNA mutations and changes in lipid peroxidation of cellular membranes and changes in enzymatic activities.[26]

Lycopene a powerful antioxidant obtained from tomatoes. It is manufactured by the Lyc-O-Mato ${ }^{\mathrm{TM}}$ process and retains its natural proportions with other compounds in the marketed pharmacological preparations. It has been shown to inhibit various types of cancers and has been shown to have potent benefits in oral premalignant lesions such as leukoplakia, where it has been shown to modulate dysplastic changes. In a recent study it is found to have shown positive result in the treatment of OSF.[27]

$16 \mathrm{mg}$ of lycopene daily in 2 divided doses for 2 months or in combination with intralesional injections of betamethasone has shown marked improvement in mouth opening and associated symptoms. [17]

Beta-carotene: topical application improves the integrity of oral epithelium as well as to induce the redifferentiation of dysplastic epithelium. Regular intake of beta-carotene combined with routine measures will considerably reduce the risk of malignant transformation.[28] 6 weeks of treatment with tablets containing mostly beta-carotene and vitamin $\mathrm{E}$ thrice daily, showed an effective increase in mouth opening and tongue protrusion in OSF patients.[29]

Alpha- Lipoic Acid also called as thioctic acid is a sulphur containing substance, acts as a coenzyme in Krebs cycle in decarboxylation of pyruvate and alpha- keto acids claimed to be the near-perfect antioxidant. It has a good potential action of scavenging free radicals and also it has excellent property, it can dissolve in both water and fat. Alpha-lipoic acid $100 \mathrm{mg}, 1$ capsule per day for 30 days has shown reduction in burning sensation and improved mouth opening. [17]

Antoxid tablet (containing beta-carotene $50 \mathrm{mg}$, Vitamin A palmitate $2500 \mathrm{IU}$, Vitamin E acetate 10 IU along with Vitamin C, zinc, manganese and copper) given thrice daily for 6 weeks has been shown to cause significant clinical improvement in patients with oral submucous fibrosis.[30]

\subsubsection{Alternative Medicine Immunised milk}

Immunised milk is a type of skim milk produced from cows immunised with multiple human intestinal bacteria. It has good anti-inflammatory effect and contains modest amounts of Vitamin A, C, B1, B2, B6, B12, nicotinic acid, pantothenic acid, folic acid, iron, copper and zinc. Though chemically it is identical to commercial milk, but it contains $20-30 \%$ higher concentration of IgG type I antibody. Anti inflammatory component can suppress the inflammatory reaction and modulate cytokine production. Relief in patients may be partially attributed to the micronutrients contained in the immune milk powder.[22]

Tai et al advocated $45 \mathrm{gms}$ of immunised milk powder twice a day, for 3 months and observed significant improvement in symptoms of OSF.[31]

\section{HERBAL EXTRACTS Turmeric}

Turmeric (haldi), a rhizome of Curcuma longa, is a flavourful yellow-orange spice. An orange pulp contained in the rhizome constitutes the source of turmeric medicinal powder. Components of turmeric are named curcuminoids, which include mainly curcumin (diferuloyl methane), demethoxycurcumin, and bisdemethoxycurcumin.[32]

Curcumin inhibits the products of inflammation such as prostaglandin and leukotreines by the way of inhibiting both cycloxygenase and lypoxygenase pathways of inflammation. Also, scavenging effect of curcumin on superoxide radicals, hydroxyl radicals and lipid peroxidation has been demonstrated. Furthermore, curcumin has been found to have fibrinolytic action which could be attributed to inhibition of lipid peroxidation, checking cellular proliferation and inhibition of collagen synthesis. 
It offers protection against benzopyrene induced increase in micronuclei in circulating lymphocytes and an excellent scavenger of free radical. Turmeric oil \& turmeric oleoresin both act synergistically in vivo to offer protection against DNA damage.[33]

Hastak et al. studied the effect of turmeric oil $(600 \mathrm{mg})$, alcohol extracts of turmeric (3g) and turmeric oleoresin $(600 \mathrm{mg})$ on cytogenetic damage in patients suffering from OSF after daily intake for 3 months. They concluded that turmeric oil and turmeric oleoresin both act synergistically in vitro to offer protection against DNA damage. Thus, curcumin holds a promising future in the treatment of OSF.[33]

Alcoholic extracts of turmeric $(3 \mathrm{~g})$, turmeric oil $(600 \mathrm{mg})$, turmeric oleoresin $(600 \mathrm{mg})$ daily for 3 months.

Aloe vera

Aloe vera is an emollient resin and a mannoprotein containing many amino acids, called 'wound healing hormones'. The polysaccharides contained in the gel of the leaves has wound healing, antiinflammatory, anticancer, immunomodulatory, and gastro protective properties. Mechanism of action is through reduceing oral symptoms like burning sensation and gradual recovering of mouthopening. It is relatively safe and can be applied topically in the treatment for OSF.

Topical application of $5 \mathrm{mg}$ of aloe vera thrice daily for three months has been reported to reduce burning sensation and improve mouth opening.[34]

Oxitard

Oxitard contains the extracts of Magnifera indica, With ania somnifera, Daucus carota, Glycyrrhiza glabra, Vitis vinifera, Syzygium aromaticum, powders of Emblica officinalis and Yashada bhasma; and oils of Triticum sativum. Singh et al used Oxitard capsule in 48 cases, with patients having difficulty in mouth opening and pain in the mouth. A dose of 2 capsules twice daily for a period of 3 months was standardized; they concluded that there was significant increase in mouth opening along with decrease in pain.[35]

Spirulina

Spirulina is blue, green algae with a rich natural source of proteins, carotenoids and other micronutrients. It has a antioxidant property with high amount of beta carotene and superoxide dismutase so it can be effectively used in treatment of OSF.[36]

\section{Combination therapy}

Significantly better results have been obtained by giving local injections of chymotrypsin, hyaluronidase and dexamethasone together than with one drug alone or a combination of dexamethasone with either chymotrypsin or hyaluronidase.[16]

Combined therapy with nylidrin hydrochloride, vitamins D, E and B complex, iodine, placental extract, local and systemic corticosteroids, and physiotherapy claims a success rate of $62 \%$ in OSF.[20]

\section{ULTRASOUND}

Ultrasound has been used extensively in physical medicine with considerable success. During ultrasound therapy, cell membrane permeability is increased by altering sodium and potassium ion gradients. This increased permeability improves gas exchange and promotes healing. It increases vasodilatation, accelerates lymph flow, decreases inflammation and stimulates metabolism. Ultrasound used for therapeutic purpose has a frequency of about $0.8-1 \mathrm{MHz}$ and an intensity of $0.5-3 \mathrm{w} / \mathrm{cm}^{2}$. Ultrasound treatment accelerate healing, increase the extensibility of collagen fibers, provide pain relief and selectively raises the temperature in some well circumscribed areas.[14,22]

Ultrasound of dose ranging from 0.6 to $2.0 \mathrm{~W} / \mathrm{Cm}^{2}$ pulsed $1: 1$ or $1: 2$ ( $50 \%$ or $33.3 \%$ duty cycle), Frequency of $3 \mathrm{MHz}, 5 \mathrm{~cm}$ diameter transducer head for 3 to 4 minutes to each side over the cheek for 15 consecutive days with permissible one day off each week.[14]

\section{STEM CELL THERAPY}

Recently scientists have proven that intralesional injection of autologous bone marrow stem cells is a safe and effective treatment modality in OSF. It has been shown autologous bone marrow stem cell injections induces angiogenisis in the area of lesion which in turn decreases the extent of fibrosis thereby leading to significant increase in mouth opening.[37]

\section{Conclusion}

OSF is one of the most poorly understood and unsatisfactorily treated diseases. Among the above mentioned modalities for treatment of OSF none of them can be considered to be completely effective.

All available treatments provide symptomatic relief, which is short lived. This is mainly due to the fact that the etiology of the disease is not fully understood and the disease is progressive in nature.

The wide range of treatment modalities currently used for OSF is ample evidence that there is no simple answer to how it should be managed. An evidence based practice will help to provide the best 
possible treatment based on sound evidence, so high-quality randomized controlled trials should be initiated and clinicians should encourage their patients to participate in clinical trials, where they have the chance to try new therapeutic agents.

\section{References}

[1] Warnakulasuriya S, Johnson NW, Waal I. Nomenclature and classification of Potentially malignant disorders of the oral mucosa. J Oral Pathol Med 2007;36:575-80.

[2] Pindborg JJ, Sirsat SM. Oral submucous fibrosis. Oral Surg Oral Med Oral Pathol 1966;22:764-79.

[3] Rajendran R, Oral sub mucous fibrosis. J Oral Maxillofac Path.2003, Jan-Jun; 7(1):1-4.

[4] Prabhu SR, Wilson DF, Daftary DK, Johnson Oral diseases in tropics. $1^{\text {st }}$ edition,p:417- 422.

[5] Ranganathan K, Devi M U, Joshua E, Kirankumar K, TSaraswathi T R. Oral submucous fibrosis: a case-control study in Chennai, South India. 2004; 33(5):274-77.

[6] Paissat DK. Oral submucous fibrosis. Int J Oral Surg 1981;10:307-12.

[7] Ariyawardana A, Panagoda G. J, Fernando, H.N, Ellepola, A. N. B, Tilakaratne, W. M, Samaranayake, L. P. Oral submucous fibrosis and oral yeast carriage-a case control study in sri lankan patients [Electronic version]. Mycoses,2007. 50(2), 116-120.

[8] Kiran K, Saraswathi, T. R, Ranganathan, K, Uma, D. M, Joshua, E. (2007). Oral submucous fibrosis: A clinico-histopathological study in chennai [Electronic version]. Indian Journal of Dental Research, 18(3), 106-111.

[9] Sirsat SM, Khanokar V R .Submucous fbrosis of the palate in diet-preconditioned Wistarrats. The Saudi Dental Journal, Volume 1, Number 2,1989 Arch Pathol 1960;70:171-179.

[10] Asnani S, Mahindra U, Oswal R. Use of diode lasers in treatment of oral submucous fibrosis: A new concept in surgical management. International Journal of Case Reports and Images. 2014: 5(3): 198-201.

[11] Ali FM, Prasant MC,Patil A, Aher V, Tahsildar S, Deshpande R. Oral Submucous Fibrosis: Medical Management. Global Journal of Medicine and Public Health. 2012;1(1): 12-18

[12] Vijayavel. T, Ponni V. mAnAgement for orAl submucous fibrosis - A comprehensive review. Indian Journal of Multidisciplinary Dentistry. 2014; 4(1): 869-874.

[13] Thakur N: Effectiveness of Micronutrients and Physiotherapy in the Management of Oral Submucous Fibrosis. Int Journal of contemporary Dentistry2011;2(1): 101-105.

[14] Vijayakumar M, Priya D. Physiotherapy for improving mouth opening \& tongue protrution in patients with Oral Submucous Fibrosis (OSMF) - Case Series. International Journal of Pharmaceutical Science and Health Care. 2013;2(3): 50-58.

[15] Madalli V, Basavaraddi S, Burde K, Horatti P. Oral Submucous Fibrosis- An Overview. International Journal of Dental Research \& Development. 2014; 4(2): 1-16.

[16] Gupta D, Sharma SC. Oral submucous fibrosis- A new treatment regimen. Oral Maxillofac Surg. 1988; 46: 830-833.

[17] Shetty DN, Shenai P, Chatra L. Oral Submucous Fibrosis: Review of Modalities of Management. Indian Dentist Research and Review.2010;66-70.

[18] Chole RH, Gondivkar SM, Gadbail AR, Balsaraf S, Chaudhary S, Dhore SV, Ghonmode S, Balwani S, Mankar M, Tiwari M, Parikh RV. Review of drug treatment of oral submucous fibrosis. Oral Oncol. 2012;48(5):393-8.

[19] Rajendran R, Rani V, Shaikh S. Pentoxifylline therapy: A new adjunct in the treatment of oral submucous fibrosis. Indian Journal of Dental Research.2006;17(4):190-198.

[20] Sharma J K, Gupta AK, Mukija RD, Nigam P. Clinical experience with the use of peripheral vasodilator in oral disorders. Int J Oral Maxillofac Surg.1987;16: 695- 9.

[21] Katharia S K, Singh S P, Kulshreshtha V K. The Effects Of Placenta Extract In Management Of Oral Submucous Fibrosis. Indian Journal of Pharmacology.1992; 24: 181-183.

[22] Taneja L., Nagpal A., Vohra P. Arya V. Oral submucous Fibrosis: An oral physician approach. J Innov Dent. 2011. 1(3)

[23] Haque M F, Meghji S, Nazir R, Harris M. Interferon gamma (IFN- $\gamma$ ) may reverse oral submucous fibrosis. J Oral Pathol Med. 2001; 30: 12-21.

[24] Kakar P.K., Puri R.K. and Venkatachalam V.P. Oral submucous fbrosis-treatment with hyalase J LaryngolOtol, 1985, 99:57-59.

[25] Jiang X, Hu J. Drug treatment of oral submucous fibrosis: a review of the literature. J Oral Maxillofac Surg. 2009 Jul;67(7):1510-5

[26] Hennekens CH. Micronutrients and cancer prevention.Cancer Det Prev. 1984; 7:147-58.

[27] Abhinav kumar et al, Oral Surg Oral Med Oral Pathol Oral Radiol Endod 2007;103:207-213

[28] Thriveni R, Praveen AH, Mubeen. Effects of Natural Beta-carotene and Commercially Available Combination of Beta-carotene, Vitamin E, Vitamin C and nerals: A Prospective Comparative Study. Journal of Indian Academy of Oral Medicine and Radiology, October-December 2011;23 (4): 554-58.

[29] Tadakamadla J, Kumar S, Mamatha GP. Non-invasive management of Oral submucous fibrosis: A Review. J Oral Health Res; January $2011,2(1)$

[30] Borle RM, Borle SR. Management of oral submucous fibrosis: A conservative approach. J Oral Maxillofac Surg. 1991;49:788-791.

[31] Tai YS, Liu BY, Wang JT, Sun A, Kwan HW, Chiang CP. Oral administration of milk from cows immunized with human intestinal bacteria leads to significant improvements of symptoms and signs in patients with oral submucous fibrosis. J Oral Pathol Med. 2001; 30: 618-625.

[32] Nagpal M and Sood S. Role of curcumin in systemic and oral health: An overview. J Nat Sci Biol Med. 2013 Jan-Jun; 4(1): 3-7

[33] Hastak K, Lubri N, Jakhi S.D. Effect of turmeric oil and turmeric oleoresin on cytogenic damage in patients suffering from oral submucous fbrosis. Cancer Letters; 116: 265-9,1997.

[34] Sudarshan R, Annigeri R G, Vijayabala G S. Aloe vera in the treatment for oral submucous fibrosis - a preliminary study. J Oral Pathol and Med.2012. 41(10):755-761.

[35] Patil S, Santosh B S, Maheshwari S, Deoghare A, Chhugani S, Rajesh P R. Efficacy of oxitard capsules in the treatment of oral submucous fibrosis. J Can Res Ther [Epub ahead of print] [cited 2014 Sep 12].

[36] Mulk BS, Deshpande P, Velpula N, Chappidi V, Chintamaneni RL, Goyal S. Spirulina and pentoxyfilline - a novel approach for treatment of oral submucous fibrosis. J Clin Diagn Res. 2013 Dec;7(12):3048-50.

[37] Sankaranarayanan S, Padmanaban J, Ramachandran CR, Manjunath S, Baskar S, Senthil Kumar R, Senthil Nagarajan R, Murugan P, Srinivasan V, Abraham S (June 2008). "Autologous Bone Marrow stem cells for treatment of Oral Sub-Mucous Fibrosis - a case report". Sixth Annual Meeting of International Society for Stem Cell Research (ISSCR), Philadelphia, PA USA. 